\title{
messemer \\ Research using autologous cord blood - time for a policy change
}

Michael X Han Honours Student (Medicine)

Maria E Craig MB BS, PhD, FRACP Paediatric Endocrinologist

1 School of Women's and Children's Health, University of New South Wales Sydney, NSW.

2 Institute of Endocrinology and Diabetes, Children's Hospital at Westmead, Sydney, NSW.

m.craig@unsw.edu.au

doi: 10.5694/mjal2.10835

Previously in this series

"Ethics series - 2" in MJA 2013; 198: 496-502

Series Editor Dominic JC Wilkinson MBBS, DPhil, FRACP $\mathrm{t}$ is now well established that type 1 diabetes is a chronic, multifactorial disease that results from autoimmune-mediated destruction of pancreatic $\beta$ cells. However, no intervention has successfully prevented the disease to date. Recently, reinfusion of autologous umbilical cord blood has been proposed as a novel preventive therapy and is the focus of an Australian Phase I trial, the Cord Reinfusion in Diabetes (CoRD) pilot study (https:// www.anzctr.org.au/Trial/Registration/TrialReview.aspx?id= 363694). However, the use of publicly stored cord blood for research in Australia is currently limited by policy that restricts its use to recognised indications, including allogeneic haematopoietic stem cell transplantation for oncological, haematological, genetic and immunological disorders. There are also specific ethical issues associated with the collection and storage of cord blood, including storage (public $\mathrm{v}$ private), informed consent (from whom, when and how?), ownership (does it belong to the child or the parent?), access (exclusive autologous v allogeneic use) and the principle of beneficence. ${ }^{1}$

A substantial body of research in recent years has been directed towards prevention of type 1 diabetes. Primary prevention has largely targeted putative environmental risk factors such as early introduction of docosahexaenoeic acid or dietary cow milk protein. ${ }^{2}$ The latter is being investigated in the international randomised controlled Trial to Reduce the Incidence of Type 1 Diabetes in the Genetically at Risk (TRIGR). ${ }^{3}$ In contrast, secondary prevention trials have targeted immunomodulation through interventions such as nicotinamide and oral or parenteral insulin. While previous trials have failed to demonstrate significant therapeutic benefit, ${ }^{2}$ and evidence-based guidelines state that no interventions are recommended for use in clinical practice to delay or prevent the onset of type 1 diabetes, ${ }^{4}$ the role of intranasal insulin is under investigation through the Type 1 Diabetes Prevention Trial. ${ }^{5}$ Nevertheless, there is a compelling argument to explore novel approaches to the prevention of type 1 diabetes.

An alternative preventive strategy is to modify the regulatory components of the immune system - in particular, Foxp3+ regulatory $\mathrm{T}$ cells (Tregs). There is emerging evidence in animal models and in humans to suggest that the loss of normal immunological selftolerance in type 1 diabetes, a crucial step in its pathogenesis, may be attributable to the failure of Tregs. ${ }^{6}$ The specific Treg abnormalities involved in type 1 diabetes are yet to be fully elucidated, but may include defects in Treg number and function, and increased resistance to regulation by effector T cells. ${ }^{6}$

\begin{abstract}
Sunnaner
- Type 1 diabetes results from the loss of normal immunological self-tolerance, which may be attributable to the failure of Foxp3+ regulatory T cells (Tregs). Umbilical cord blood is rich in Tregs and therefore has the potential to prevent or delay the onset of type 1 diabetes. A pilot trial is currently underway in Australia to examine whether infusion of autologous cord blood can prevent type 1 diabetes in high-risk children with serum antibodies to multiple $\beta$-cell antigens.

- A number of other potential therapeutic indications for autologous cord blood have been proposed, including cerebral palsy and hypoxic-ischaemic encephalopathy.

- Recruitment to clinical trials using cord blood is influenced by divergent public and private cord blood banking policy in Australia. The burgeoning consumer demand for storage of cord blood highlights the need for regulatory bodies to develop and adapt policies to facilitate research that may extend the use of cord blood beyond currently recognised indications.

- Consumers, researchers and policymakers must also recognise specific ethical issues associated with collection and storage of cord blood, including storage in public and private banks, informed consent, ownership, access and the principle of beneficence.
\end{abstract}

Umbilical cord blood is rich in Tregs, which become functionally suppressive on antigen stimulation, ${ }^{7}$ and is also a source of haematopoietic and pluripotent stem cells. ${ }^{8}$ Thus, there is a strong scientific rationale behind the potential for cord blood to prevent or delay the onset of type 1 diabetes. The CoRD trial will examine whether autologous cord blood infusion can prevent type 1 diabetes in high-risk children with serum antibodies to multiple $\beta$-cell antigens. In parallel, the trial will study the immunological effects of cord blood infusion. This is the first time such a trial will be undertaken for the prevention of type 1 diabetes in humans, although studies have used autologous cord blood after the onset of type 1 diabetes. In a Phase I trial involving 24 children (median age, 5.1 years) with type 1 diabetes, ${ }^{9}$ infusion of autologous cord blood after a median diabetes duration of 3 months was associated with a transient increase in total and naive Tregs at 6 and 9 months, respectively. No adverse events were observed. Nevertheless, the intervention did not preserve $\beta$-cell function, as C-peptide levels decreased after infusion. However, the time after diagnosis at which the infusion was given may have been important; a rapid loss of $\beta$-cell mass has been frequently observed, and this decline may have occurred before the infusion was given. In a pilot study of 15 individuals (median age, 29 years) with type 1 diabetes, ${ }^{10}$ circulating lymphocytes were cocul- 
tured with allogeneic cord blood-derived stem cells and subsequently reintroduced into the circulation. There was a significant improvement in mean fasting C-peptide levels and a reduction in glycated haemoglobin levels and daily insulin requirements, in parallel with an increase in Tregs. The procedure was well tolerated, with no adverse events. These two studies suggest that cord blood may increase the frequency of Tregs in people with type 1 diabetes and may therefore induce immune tolerance. Whether cord blood has the same effect among people with prediabetes is unknown.

There are several fundamental methodological issues that must be addressed in the development of trials such as CoRD, which involve autologous cord blood. Studies that have demonstrated either no or minimal adverse effects in the use of autologous cord blood have involved small study samples. ${ }^{9,10}$ While the safety of autologous cord blood may also be inferred from the known safety of allogeneic cord blood, further data are required, particularly in the paediatric population. Rates of microbial contamination are low ( $<5 \%$ in privately banked samples), although such samples are generally not suitable for use. In addition, samples with low total nucleated cell counts may be ineffective; ${ }^{11}$ however, private banks specify a lower limit of $10^{8}$ total nucleated cells for storage, thereby reducing the likelihood of inadequate samples being collected.

Despite the clear need for well designed trials to examine the specific therapeutic applications of cord blood, there are important differences in the ways in which public and private banks collect, store and provide access to cord blood, which could affect potential research. Public banks store donated cord blood units for allogeneic use, with around 3000 units stored per year in Australia (about 1\% of live births). The rate of collection in public banks is dependent on available funding and only a few hospitals participate in collection nationally. In contrast, private banks provide storage for personal and familial use, for a fee. The storage rate in private banks is around 4000 units per year (Mark Kirkland, Cord Blood Bank Director, Cell Care Australia, personal communication), and growth is estimated at $12 \%-15 \%$ per annum. ${ }^{12}$ Globally, over a million cord blood units have been stored in private banks. Nevertheless, the chance of using a privately stored cord blood sample is less than $0.01 \% .{ }^{13}$ Although a number of potential therapeutic indications for autologous cord blood have been proposed - such as cerebral palsy, hypoxicischaemic encephalopathy, ${ }^{14}$ congenital hydrocephalus and stroke $e^{15}$ - there are few published data. The number of published clinical trials using autologous cord blood is limited; however, there are 14 ongoing trials registered on ClinicalTrials.gov using both publicly and privately stored cord blood. ${ }^{16}$

The expansion of cord blood trials, along with high consumer demand for storage, places pressure on regulatory bodies to develop and adapt policies to meet these needs. Although the regulatory framework surrounding cord blood banking in Australia has undergone significant development, issues remain regarding access to publicly donated cord blood. In particular, there is no clear guideline that addresses degifting and use of publicly stored cord blood for autologous reinfusion beyond recognised indications. At present, the use of publicly banked cord blood is essentially limited to well researched and established applications, particularly for haematopoietic reconstitution, and does not extend to research purposes (Anthony Montague, National Cord Blood Network Operations Manager, Australian Bone Marrow Donor Registry, personal communication). These processes are, however, currently under review. Beyond being a policy issue, this raises deeper ethical questions regarding the rights of public donors to access their donated cord blood and equity between public donors and those who privately bank cord blood, particularly as the private industry continues to expand. ${ }^{17,18}$

While the future applicability of cord blood-based therapeutics, including prevention of type 1 diabetes, is at present unclear, this is an emerging area of research. An evidence base is clearly needed in response to the burgeoning interest in the community for storage of cord blood. However, important questions regarding the storage and use of publicly donated cord blood remain unanswered. Should cord blood banks be permitted to degift altruistically donated samples to enable participation in research? Will novel therapeutic uses for cord blood lead to changes to public cord blood banking policy? Given the likelihood of future cord blood-based clinical trials, the existing framework of cord blood banking policy must be reviewed to meet the needs that will be posed by such research, which may lead the way to expanding novel uses of cord blood.

Competing interests: No relevant disclosures.

Provenance: Not commissioned; externally peer reviewed.

1 Petrini C. Umbilical cord blood collection, storage and use: ethical issues. Blood Transfus 2010; 8: 139-148.

2 Gallagher MP, Goland RS, Greenbaum CJ. Making progress: preserving beta cells in type l diabetes. Ann NY Acad Sci 2011; 1243: 119-134.

3 Akerblom HK, Krischer J, Virtanen SM, et al; The TRIGR Study Group. The Trial to Reduce IDDM in the Genetically at Risk (TRIGR) study: recruitment, intervention and follow-up. Diabetologia 2011; 54: 627-633.

4 Craig ME, Twigg SM, Donaghue KC, et al; Australian Type 1 Diabetes Guidelines Expert Advisory Group. National evidence-based clinical care guidelines for type 1 diabetes in children, adolescents and adults. Canberra: Australian Government Department of Health and Ageing, 2011.

5 Stop Diabetes Australia. Type 1 Diabetes Prevention Trial. http://www. stopdiabetes.com.au (accessed Jun 2013).

6 Battaglia M, Roncarolo MG. Immune intervention with T regulatory cells: past lessons and future perspectives for type I diabetes. Semin Immunol 2011; 23: 182-194.

7 Mayer E, Bannert C, Gruber S, et al. Cord blood derived CD4+ CD25(high) T cells become functional regulatory T cells upon antigen encounter. PLOS One 2012; 7: e29355.

8 Rao M, Ahrlund-Richter L, Kaufman DS. Concise review: cord blood banking, transplantation and induced pluripotent stem cell: success and opportunities. Stem Cells 2012; 30: 55-60.

9 Haller MJ, Wasserfall CH, Hulme MA, et al. Autologous umbilical cord blood transfusion in young children with type 1 diabetes fails to preserve C-peptide. Diabetes Care 2011; 34: 2567-2569.

10 Zhao Y, Jiang Z, Zhao T, et al. Reversal of type 1 diabetes via islet beta cell regeneration following immune modulation by cord blood-derived multipotent stem cells. BMC Med 2012; 10: 3 .

11 Rosenau EH, Sugrue MW, Haller M, et al. Characteristics of thawed autologous umbilical cord blood. Transfusion 2012; 52: 2234-2242.

12 Mayani H. Umbilical cord blood: lessons learned and lingering challenges after more than 20 years of basic and clinical research. Arch Med Res 2011; 42: 645-651.

13 Rosenthal J, Woolfrey AE, Pawlowska A, et al. Hematopoietic cell transplantation with autologous cord blood in patients with severe aplastic anemia: an opportunity to revisit the controversy regarding cord blood banking for private use. Pediatr Blood Cancer 2011; 56: 1009-1012.

14 Jensen A, Hamelmann E. First autologous cell therapy of cerebral palsy caused by hypoxic-ischemic brain damage in a child after cardiac arrest-individual treatment with cord blood. Case Rep Transplant 2013; 2013 : 951827. doi: 10.1155/2013/951827.

15 Wang F, Maeda N, Yasuhara T, et al. The therapeutic potential of human umbilical cord blood transplantation for neonatal hypoxic-ischemic brain injury and ischemic stroke. Acta Med Okayama 2012; 66: 429-434.

16 US National Institutes of Health. ClinicalTrials.gov. http://clinicaltrials.gov/search?intr= autologous+cord+blood (accessed Aug 2013).

17 Sugarman J, Kaalund V, Kodish E, et al. Ethical issues in umbilical cord blood banking. JAMA 1997; 278: 938-943. 


\section{Ethics and law}

18 Burgio GR, Gluckman E, Locatelli F. Ethical reappraisal of 15 years of cord-blood

transplantation. Lancet 2003; 361: 250-252. 